\title{
Community structure of shallow rocky shore fish in a tropical bay of the southwestern Atlantic
}

\author{
Camilo Moitinho Ferreira ${ }^{1}$, Ericka O. Cavalcanti Coni ${ }^{1}$, Diego V. Medeiros ${ }^{2}$, Cláudio L. S. Sampaio ${ }^{3}$, \\ José Amorim Reis-Filho ${ }^{4}$, Francisco Barros ${ }^{5}$, Miguel Loiola ${ }^{6}$, José de Anchieta Cintra da Costa Nunes ${ }^{4, *}$ \\ ${ }^{1}$ Programa de Pós Graduação em Ecologia e Conservação, Universidade Estadual da Paraíba. Instituto de Gestão, Estudos e \\ Monitoramento Ambiental (IGEMA). Ecology \& Environmental Science, School of Biological Sciences, University of Adelaide, South \\ Australia, SA 5005, Australia. \\ (Av. das Baraúnas, 351 - Campus Universitário, CEP: 58109-753, Campina Grande, PB, Brazil. Rua Eduardo Pinto, no 49, \\ CEP: 42835-000, Arembepe, Camaçari, Bahia, Brazil. Southern Seas Ecology Laboratories, room 128b, Darling Building, DX 650 418). \\ ${ }^{2}$ Programa de Pós Graduação em Ecologia e Monitoramento Ambiental, Universidade Federal da Paraíba. \\ (Rua da Mangueira, CEP: 58297-000 Rio Tinto, PB, Brazil). \\ ${ }^{3}$ Universidade Federal de Alagoas, Polo Penedo, Departamento de Engenharia de Pesca. Laboratório de Ictiologia e Conservação (LIC). \\ (Av. Beira Rio s/no ${ }^{\circ}$ Centro Histórico, CEP: 57200-000, Penedo, Alagoas, Brazil). \\ ${ }^{4}$ Laboratório de Ecologia Bentônica (LEB), Universidade Federal da Bahia. Programa de Pós Graduação em Ecologia e \\ Biomonitoramento, Universidade Federal da Bahia. Instituto de Educação, Ciência e Utilidade Sócio-Ambiental (ECUS). \\ (Rua Barão de Geremoabo, s/n Ondina, CEP: 40170-115, Salvador, BA, Brazil. Rio Vermelho, CEP: 41940-250, Salvador, Bahia, Brazil). \\ ${ }^{5}$ Universidade Federal da Bahia, Laboratório de Ecologia Bentônica. \\ (Rua Barão de Jeremoabo, s/n Ondina, CEP: 40170-115, Salvador, BA, Brazil. Rio Vermelho, CEP: 41940-250, Salvador, Bahia, Brazil). \\ ${ }^{6}$ Laboratório de Estudos de Recifes de Corais e Mudanças Globais (RECOR), Universidade Federal da Bahia. \\ (Rua Barão de Jeremoabo, CEP: 40170-115, Salvador, BA, Brazil. Rio Vermelho, CEP: 41940-250, Salvador, Bahia, Brazil).
}

*Corresponding author: anchietanunesba@gmail.com

\section{Abstract}

Southwestern Atlantic Ocean rocky shores sustain important reef fish communities. However, those communities in tropical regions are not well understood, especially in Brazil. In this present article we assess community parameters of reef fishes such as composition, trophic organization and their relationships with physical and biological factors on four tropical rocky shores in Todos os Santos Bay, southwestern Atlantic. During six months, a total of 80 visual censuses were performed, in which 3,582 fish belonging to 76 species were recorded. Territorial herbivorous fish and turf algae were dominant at all the sites. The spatial variability of fish community structure was related to the benthic cover composition and depth. The high abundance of territorial herbivores and mobile invertebrate feeders could be associated with high levels of turf cover, low wave exposure and shallow waters. Moreover, this fact could be a consequence of the low density of roving herbivores and large carnivores probably due to the pressure of intense fishing activity. Thus complementary studies are needed to evaluate the actual conservation status of these rocky shore reefs, singularly located habitats connecting inner and outer reefs in Todos os Santos Bay.

Descriptors: Todos os Santos Bay, Rocky shores, Fish communities, Habitat structure, Trophic groups, Visual census.

\section{RESUMo}

Os costões rochosos do Atlântico Sul Ocidental sustentam ricas comunidades de peixes recifais. Apesar disso, o conhecimento sobre essas comunidades em zonas tropicais continua escasso, especialmente no Brasil. No presente trabalho avaliamos parâmetros das comunidades de peixes recifais, tais como composição e estrutura trófica, além de sua interação com variáveis físicas e bióticas, em quatro costões rochosos tropicais na Baía de Todos os Santos, costa leste do Brasil. Durante seis meses 80 censos visuais foram realizados, nos quais 3.582 peixes pertencentes a 76 espécies foram registrados. Os peixes herbívoros territoriais e as algas filamentosas dominaram em todos os costões. A variação espacial na estrutura da comunidade de peixes esteve relacionada com a composição bêntica e profundidade. A elevada abundância observada de peixes herbívoros territoriais e invertívoros móveis pode ser devido à alta cobertura de algas filamentosas e à baixa profundidade e exposição às ondas. Além disso, esse padrão também pode ser explicado pela baixa densidade de herbívoros errantes e grandes carnívoros, provavelmente devido à intensa pressão da atividade pesqueira. Assim, novos estudos são necessários para avaliar o real estado de conservação destes ambientes, uma vez que estão localizados em posição singular na Baía de Todos os Santos, conectando recifes internos e externos da área.

Descritores: Baía de Todos os Santos, Costões rochosos, Comunidades de peixes, Estrutura trófica, Censos visuais. 


\section{INTRODUCTION}

Coastal environments, such as coral reefs and rocky shores are amongst the most diverse and productive ecosystems in the world (PAULY; CHRISTENSEN, 1995; REAKA-KUDLA, 1997). Besides their ecological relevance, reef systems are also economically important for thousands of people living in tropical coastal areas (RUSS, 1991; COSTANZA et al., 1997; MOBERG; FOLKE, 1999; RUSS; ALCALA, 1999). However, all these environments are in a critical state of degradation, mainly due to natural and anthropogenic factors such as global climate change, marine pollution, overfishing and unplanned coastal development (HUGHES, 1994; DUFFY, 2003; PANDOLFI et al., 2003; BELLWOOD et al., 2004; WILKINSON, 2008; BRUCE et al., 2012; FRANCINI-FILHO et al., 2008).

The Brazilian reef environments encompass oceanic reef systems, such as the archipelagoes of Fernando de Noronha, St. Peter and St. Paul, Trindade/Martim Vaz (rocky islands) and the Rocas Atoll (a biogenic island), as well as coastal reef systems (FLOETER; GASPARINI, 2000). The country's eastern and northeastern coastlines primarily present coral reef structures, while the southern and southeastern regions are predominated by rocky shore structures (FLOETER et al., 2006). Rocky shores are considered coastal environments formed by rock outcrops located in the transitional zone between terrestrial and aquatic environments, in which the submerged part harbors complex marine life, including animals and plants of various phyla (FERREIRA et al., 2001). Compared to coral reefs, Brazilian rocky shores show relatively low structural complexity. However, their high species richness and reef fish diversity are well recognized (FERREIRA et al., 2001; HOSTIM-SILVA et al., 2006; RANGEL et al., 2007; CHAVES; MONTEIRO-NETO, 2009).

The Brazilian northeastern reef systems (e.g. rocky and coral reefs) are considered "hotspots" of biodiversity due to the high occurrence of endemic and threatened species, particularly coral and fish fauna (LEÃO et al., 2003; MOURA, 2003). However, the majority of reef fish community structure studies have been performed on coral reef systems (FERREIRA et al., 2001; NUNES et al., 2013). Knowledge about rocky shores in tropical regions of Brazil is insufficient, especially considering their fauna and flora in the northern and northeastern regions. Such data deficiency hampers the creation and implementation of effective conservation and management policies.
Several studies have shown habitat complexity and reef fish community associations (e.g. LUCKHURST; LUCKHURST, 1978; FERREIRA et al., 2001; WILSON et al., 2007; FRANCINI-FILHO; MOURA, 2008; MEDEIROS et al., 2011; PEREIRA-FILHO et al., 2011; CONI et al., 2013). Topographic complexity is an important factor that is generally positively associated with reef fish species richness and diversity, particularly as it provides shelter from predators (HIXON; BEETS, 1993). Additionally, it may also modify the competition, interaction and survival dynamics within the community (JONES, 1988; GUNST, 2002; FRANCINI-FILHO et al., 2010). In southeastern Brazil, the habitat complexity of rocky shores is primarily determined by the presence or absence of crevices, a consequence of the superposition of rocks (FERREIRA et al., 2001). To date, there are no studies that evaluate the relationship between reef fish communities and the biotic and physical variables of rocky shores in northeastern Brazil.

Here we present the reef fish species associated with the rocky shores at Todos os Santos Bay's northern entrance (near the municipality of Salvador), as well as describe the reef fish community's spatial patterns (including trophic organization, density and species richness) and the relationship between fish density and environmental variables (physical structure and benthic cover). The Todos os Santos Bay region harbors a wide variety of marine organisms and encompasses complex environments. However, studies, particularly about fish communities, are still insufficient. Given this, this study is a first effort to characterize the fish community structure off tropical rocky shores in the northeastern region of Brazil. It is believed that this will provide essential knowledge to support future decisions for conservation and management strategies in the Todos os Santos Bay, and all tropical rocky shores in the northeast of Brazil. A succinct history of conservation in these areas is also presented.

\section{MATERIAL AND METHODS}

\section{STUdy AREA}

Todos os Santos Bay is the second largest semienclosed bay in Brazil covering approximately $1,000 \mathrm{~km}^{2}$ (LESSA et al., 2000). This area harbors a high level of biodiversity, comprising distinct habitats such as coral reefs, mangrove swamps and seagrass beds (CASTRO; 
PIRES, 2001; LEÃO et al., 2003; REIS-FILHO et al., 2010; REIS-FILHO et al., 2011). The bay has a stable annual water temperature, with low temperature fluctuation varying generally between $23^{\circ}$ and $27^{\circ} \mathrm{C}$ (CIRANO; LESSA, 2007).

The present study was carried out at the mouth of Todos os Santos bay, located off the city of Salvador. The four rocky shores (CV - Corredor da Vitória, PB - Porto da Barra, FB - Farol da Barra, and MP - Marco Polo) (Figure 1) studied are distributed along approximately four kilometers of shore line, with an area of $\sim 0.04 \mathrm{~km}^{2}$. These are characterized as being relatively shallow (max. $10 \mathrm{~m}$ depth) with the benthic substrates composed predominantly of turf algae, macroalgae and the zoanthids Palythoa caribaeorum and Zoanthus sociatus. However, other benthic organisms, such as the black sea urchin (Echinometra lucunter), ascidians and coral colonies of Favia gravida, Agaricia agaricites, Montastraea cavernosa, Mussismilia hispida, Siderastrea spp., and the two species of fire corals Millepora alcicornis and Millepora nitida, are also found within the area. These rocky shores are heavily exploited for tourism activities, artisanal fisheries, ornamental marine organism trade and spearfishing (SAMPAIO; NOTTIGHAM, 2008; NUNES et al., 2012). Despite the water's typically remaining clear throughout the year (occasionally reaching over 40 meters of visibility), during our study horizontal visibility ranged from 5 to $20 \mathrm{~m}$.

\section{FISH COMMUNITY STRUCTURE}

Free diving was conducted on all four rocky shores between August 2007 and January 2008 to assess the structure of fish communities and benthic cover. To assess the reef fish species richness and density, 20 visual censuses were randomly performed at each site using strip transects of $30 \times 2 \mathrm{~m}$ (adapted from BROCK, 1954), to a total of 80 censuses. All the fishes observed were identified to species level in accordance with the specific literature (CARVALHO-FILHO, 1999; HOSTIM-SILVA et al., 2006). Additionally, recent changes in the classification of the Serranidae and Scaridae families were taken into consideration and adopted as proposed by CRAIG and HASTINGS (2007) and WESTNEAT and ALFARO (2005).

Then species were organized in nine trophic categories, following RANDALL (1967), FERREIRA et al. (2004), FROESE and PAULY (2014), as follows:
CAR = Carnivores (those feeding on a variety of invertebrates and/or fish), PIS = Piscivores (particularly fish feeders), SIF = Sessile invertebrate feeders (feeding primarily on sessile benthic invertebrates associated with the hard or nearby soft-substrate), MIF = Mobile invertebrate feeders (feeding on a variety of mobile benthic species), OMN = Omnivores (feeding on a variety of organisms, both animal and plant materials), PLANK = Planktivores (feeding primarily on macroand micro-zooplankton), ECTFE = Ectoparasite feeders (represented by the species Elacatinus figaro), $\mathrm{RH}=$ Roving herbivorous (large and mobile species of fish which feed primarily on a variety of algae and detritus) and $\mathrm{TH}=$ Territorial herbivorous (herbivorous fish which defend their territories against other fishes).

\section{HABITAT COMPLEXITY}

Rugosity (topographic complexity) was measured for each site as an index, using the link-chain method proposed by LUCKHURST and LUCKHURST (1978). In each transect the rugosity and depths were calculated from three measurements (taken from the beginning, middle and end of the line). To evaluate the relative benthic cover, the line-point intercept transect method was used (OHLHORST et al., 1988; ROGERS et al., 1994). The number of observations was divided by the total number of points observed, to estimate the relative coverage of benthic groups (CROSBY; REESE, 1996). A total of 480 observations were measured at each location. Only major functional groups of benthic organisms were considered in this study: macroalgae, turf algae (epilithic algae and macroalgae recruits smaller than $5 \mathrm{~mm}$ ), crustose coralline algae, scleractinian corals, fire corals, octocorals, zoanthids, sponges, ascidians, echinoderms, echionoderms and barnacles.

\section{DATA ANALYSIS}

One-Way Analysis of Variance (ANOVA) was used to evaluate spatial differences on density, trophic structure (through the density of nine trophic groups) and habitat complexity (rugosity, depth and benthic cover). To compare the fish species richness between sites, random boot-strapping permutations of the data (1000 times) were performed to calculate the mean species accumulation curves and their standard deviation (GOTELLI; COLWEL, 2001). Accumulation curves were calculated using the specaccum tool, in 


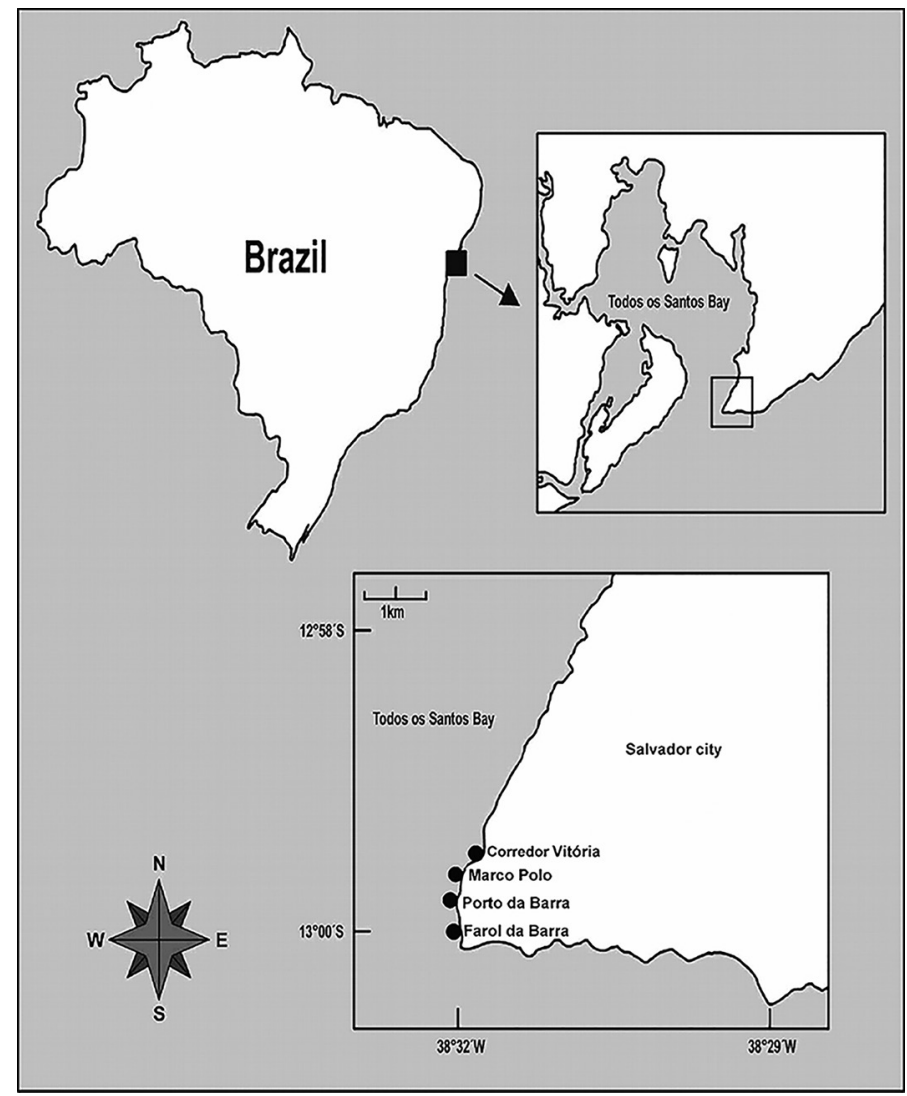

Figure 1. Map of the Todos os Santos Bay, Northeastern Brazil, showing the Rocky shores studied.

the vegan package (OKSANEN et al., 2013) of the $\mathrm{R}$ software. When significant differences were found, Student-Newman-Keuls (SNK) multiple comparisons were used as a post hoc test. Densities data were logtransformed $[\log (x+1)]$ and the relative cover was transformed to $\arcsin (\sqrt{ } \mathrm{x}+1)$ in order to meet normality and homoscedasticity assumptions (ZAR, 1999).

Multidimensional scaling (MDS) was performed based on an integrating matrix of fish fauna assemblage and benthic cover for all sampling sites. This assisted in summarizing potential similarities on the structure of fish and benthic assemblages over the space (Bray-Curtis). This method is generally the most efficient ordination method for ecological communities (MCCUNE; GRACE, 2002). Additionally, an analysis of similarity (ANOSIM), an effective test towards statistical metrics, was performed to evaluate significant differences between groups of samples corresponding to the four rocky shores sampled. A similarity perceptual test (pairwise ANOSIM) was added in order to clarify the similarity levels amongst sites. Finally, a Canonical Correspondence Analysis (CCA) was used to analyze the relationship between the fish community structure and explanatory habitat variables (rugosity, depth and benthic composition). This ordination represents a powerful technique used to understand how multiple species simultaneously respond to environmental factors, extracting significant gradients from ecological matrices (TER BRAAK; VERDONSCHOT, 1995). To increase the model power, a forward selection procedure was used to include only the most important variables. Finally, the Monte Carlo test (999 permutations) was incorporated in the final model to check if the axes were significant. Species present in less than $5 \%$ of the samples were excluded from MDS, ANOSIM, and CCA analysis. 


\section{RESULTS}

\section{COMPOSITION PATTERNS OF ROCKY SHORE FISHES}

In the present study, 3,582 fishes belonging to 76 species distributed in 35 families were registered (Table 1), averaging 0.75 individuals per $\mathrm{m}^{2}$. The species accumulation curves demonstrated that the species richness was different between the sites. Marco Polo and Porto da Barra rocky shores showed the highest species richness, followed by Corredor da Vitória and Farol da Barra (MP $>\mathrm{PB}=\mathrm{CV}>\mathrm{FB}$ ) (Figure 2).

Regarding fish density, there was a significant difference amongst sampled sites $(\mathrm{F}=2.76 ; p=0.04$; $\mathrm{SNK}$ test: $\mathrm{PB}=\mathrm{FB}>\mathrm{CV}>\mathrm{MP}$ ) with higher densities at Corredor da Vitória $\left(1.18 \pm 0.22\right.$ fish. $\left.\mathrm{m}^{-2}\right)$, followed by Porto da Barra (1.01 \pm 0.11 fish.m $\left.{ }^{-2}\right)$, Farol da Barra $(0.88 \pm 0.09$ fish. $\left.\mathrm{m}^{-2}\right)$ and Marco Polo $\left(0.65 \pm 0.05\right.$ fish. $\left.\mathrm{m}^{-2}\right)$. The ten most abundant species in decreasing order were: Stegastes fuscus, Ophioblennius trinitatis, Acanthurus bahianus, Haemulon aurolineatum, Abudefduf saxatilis, Acanthurus chirurgus, Pseudupeneus maculatus, Sparisoma axillare, Pempheris schomburgki and Labrisomus nuchipinnis. The Brazilian damselfish S. fuscus was clearly the dominating species at all the sites with its higher density found at Corredor da Vitória and Farol da Barra. Following S. fuscus, O. trinitatis was the second most dominant fish species at Porto da Barra and Farol da Barra, $H$. aurolineatum at Marco Polo and A. saxatilis at Corredor da

Table 1. Species list (Mean density (ind. $\left.\mathrm{m}^{-2}\right) \pm$ Standard error) observed in the dives, organized in phylogenetic order, according to Nelson (2006).

\begin{tabular}{|c|c|c|c|c|c|}
\hline Family & Species & Vitória & Marco Polo & Porto da Barra & Farol da Barra \\
\hline \multirow[t]{3}{*}{ MURAENIDAE } & Gymnothorax vicinus (Castelnau. 1855) & $0.001 \pm 0.001$ & $0.001 \pm 0.001$ & $0.001 \pm 0.001$ & - \\
\hline & Gymnothorax funebris Ranzani. 1840 & $0.001 \pm 0.001$ & - & $0.001 \pm 0.001$ & $0.001 \pm 0.001$ \\
\hline & Gymnothorax moringa (Cuvier.1829) & $0.001 \pm 0.001$ & $0.001 \pm 0.001$ & $0.002 \pm 0.001$ & - \\
\hline \multirow[t]{4}{*}{ OPHICHTHIDAE } & Ahlia sp. & - & - & $0.001 \pm 0.001$ & - \\
\hline & Myrichthys breviceps (Richardson. 1848) & $0.002 \pm 0.001$ & - & $0.002 \pm 0.001$ & - \\
\hline & Myrichthys ocellatus (Lesueur. 1825) & $0.001 \pm 0.001$ & - & $0.002 \pm 0.001$ & $0.001 \pm 0.001$ \\
\hline & Ophictis ophis (Linnaeus. 1758) & $0.004 \pm 0.004$ & $0.001 \pm 0.001$ & - & - \\
\hline SYNODONTIDAE & Synodus sp. & $0.003 \pm 0.002$ & $0.001 \pm 0.001$ & $0.006 \pm 0.003$ & - \\
\hline OGCOCEPHALIDAE & Ogcocephalus vespertilio (Linnaeus. 1758) & $0.001 \pm 0.001$ & $0.004 \pm 0.002$ & - & - \\
\hline \multirow[t]{2}{*}{ HOLOCENTRIDAE } & Holocentrus adscensionis (Osbeck. 1771) & $0.003 \pm 0.003$ & $0.020 \pm 0.009$ & $0.011 \pm 0.005$ & $0.002 \pm 0.002$ \\
\hline & Myripristis jacobus Cuvier. 1829 & $0.001 \pm 0.001$ & - & $0.001 \pm 0.001$ & - \\
\hline FISTULARIIDAE & Fistularia tabacaria Linnaeus. 1758 & $0.001 \pm 0.001$ & - & $0.001 \pm 0.001$ & - \\
\hline \multirow[t]{2}{*}{ SCORPAENIDAE } & Scorpaena plumieri Bloch. 1789 & - & $0.001 \pm 0.001$ & $0.001 \pm 0.001$ & $0.001 \pm 0.001$ \\
\hline & Scorpaenodes caribbaeus Meek and Hildebrand.1928 & $0.003 \pm 0.002$ & $0.001 \pm 0.001$ & - & $0.001 \pm 0.001$ \\
\hline \multirow[t]{4}{*}{ SERRANIDAE } & Rypticus saponaceus (Bloch and Schneider. 1801) & - & $0.001 \pm 0.001$ & - & - \\
\hline & Rypticus subbifrenatus (Gill. 1861) & - & - & $0.001 \pm 0.001$ & - \\
\hline & Rypticus bistrispinus (Mitchill. 1818) & $0.002 \pm 0.001$ & $0.001 \pm 0.001$ & $0.005 \pm 0.003$ & - \\
\hline & Serranus baldwini (Evermann and Marsch. 1900) & - & $0.001 \pm 0.001$ & - & - \\
\hline \multirow[t]{2}{*}{ EPINEPHELIDAE } & Cephalopholis fulva (Linnaeus. 1758) & $0.016 \pm 0.006$ & $0.008 \pm 0.004$ & $0.007 \pm 0.003$ & $0.003 \pm 0.002$ \\
\hline & Epinephelus adscensionis (Osbeck. 1765) & - & $0.001 \pm 0.001$ & $0.002 \pm 0.001$ & $0.004 \pm 0.002$ \\
\hline APOGONIDAE & Apogon sp. & - & - & $0.002 \pm 0.002$ & - \\
\hline CARANGIDAE & Caranx bartholomaei (Cuvier. 1833) & $0.003 \pm 0.003$ & - & - & $0.001 \pm 0.001$ \\
\hline GERREIDAE & Eucinostomus sp. & $0.002 \pm 0.001$ & $0.002 \pm 0.001$ & $0.004 \pm 0.003$ & $0.008 \pm 0.008$ \\
\hline \multirow[t]{6}{*}{ HAEMULIDAE } & Anisotremus surinamensis (Bloch. 1791) & - & $0.001 \pm 0.001$ & - & - \\
\hline & Anisotremus virginicus (Linnaeus. 1758) & - & $0.001 \pm 0.001$ & - & $0.001 \pm 0.001$ \\
\hline & Haemulon aurolineatum Cuvier. 1830 & $0.073 \pm 0.068$ & $0.046 \pm 0.037$ & $0.048 \pm 0.047$ & - \\
\hline & Haemulon parra (Desmarest. 1823) & $0.006 \pm 0.003$ & $0.008 \pm 0.007$ & $0.038 \pm 0.021$ & $0.006 \pm 0.005$ \\
\hline & Haemulon steindachneri (Jordan and Gilbert.1882) & $0.024 \pm 0.006$ & $0.009 \pm 0.006$ & $0.003 \pm 0.002$ & - \\
\hline & Haemulon squamipinna Rocha and Rosa. 1999 * & $0.001 \pm 0.001$ & - & - & $0.001 \pm 0.001$ \\
\hline
\end{tabular}


Continued Table 1.

\begin{tabular}{|c|c|c|c|c|c|}
\hline \multirow[t]{2}{*}{ SCIAENIDAE } & Odontoscion dentex (Cuvier. 1830) & - & - & $0.008 \pm 0.008$ & - \\
\hline & Pareques acuminatus (Bloch and Schneider.1801) & $0.034 \pm 0.031$ & $0.002 \pm 0.002$ & $0.001 \pm 0.001$ & $0.002 \pm 0.002$ \\
\hline \multirow[t]{2}{*}{ MULLIDAE } & Mulloidichthys martinicus (Cuvier. 1829) & $0.001 \pm 0.001$ & - & - & - \\
\hline & Pseudupeneus maculatus (Bloch. 1793) & $0.013 \pm 0.006$ & $0.029 \pm 0.015$ & $0.029 \pm 0.013$ & $0.042 \pm 0.024$ \\
\hline PEMPHERIDAE & Pempheris schomburgki Müller and Troschel.1848 & $0.016 \pm 0.016$ & - & $0.063 \pm 0.063$ & - \\
\hline CHAETODONTIDAE & Chaetodon striatus Linnaeus. 1758 & $0.011 \pm 0.004$ & $0.009 \pm 0.003$ & $0.020 \pm 0.006$ & $0.010 \pm 0.006$ \\
\hline \multirow[t]{2}{*}{ POMACANTHIDAE } & Holocanthus tricolor (Bloch. 1795) & - & $0.001 \pm 0.001$ & - & - \\
\hline & Pomacanthus paru (Bloch. 1787) & - & - & - & $0.001 \pm 0.001$ \\
\hline KYPHOSIDAE & Kyphosus sp. & $0.004 \pm 0.002$ & $0.001 \pm 0.001$ & $0.006 \pm 0.006$ & - \\
\hline CIRRHITIDAE & Amblycirrhitus pinos (Mowbray. 1927) & $0.002 \pm 0.001$ & $0.001 \pm 0.001$ & - & - \\
\hline \multirow[t]{5}{*}{ POMACENTRIDAE } & Abudefduf saxatilis (Linnaeus. 1758) & $0.019 \pm 0.017$ & $0.011 \pm 0.008$ & $0.019 \pm 0.017$ & $0.013 \pm 0.010$ \\
\hline & Chromis multilineata (Guichenot. 1853) & - & $0.002 \pm 0.001$ & $0.036 \pm 0.022$ & - \\
\hline & Stegastes fuscus (Cuvier. 1830) * & $0.572 \pm 0.077$ & $0.285 \pm 0.070$ & $0.271 \pm 0.022$ & $0.393 \pm 0.040$ \\
\hline & Stegastes pictus (Castelnau. 1855) * & $0.003 \pm 0.003$ & - & $0.001 \pm 0.001$ & $0.003 \pm 0.002$ \\
\hline & Stegastes variabilis (Castelnau. 1855) & - & $0.002 \pm 0.001$ & $0.007 \pm 0.003$ & $0.016 \pm 0.006$ \\
\hline \multirow[t]{9}{*}{ LABRIDAE } & Halichoeres brasiliensis (Bloch. 1791)* & $0.004 \pm 0.002$ & $0.001 \pm 0.001$ & $0.001 \pm 0.001$ & $0.003 \pm 0.002$ \\
\hline & Halichoeres penrosei (Starks. 1913) * & - & $0.004 \pm 0.002$ & $0.023 \pm 0.001$ & - \\
\hline & Halichoeres poeyi (Steindachner. 1867) & $0.020 \pm 0.006$ & $0.014 \pm 0.006$ & $0.014 \pm 0.006$ & $0.009 \pm 0.004$ \\
\hline & Thalassoma noronhanum (Boulenger. 1890) * & - & - & $0.001 \pm 0.001$ & - \\
\hline & Xyricthis novacula (Linnaeus. 1758) & - & $0.001 \pm 0.001$ & - & - \\
\hline & Sparisoma amplum (Ranzani. 1842)* & - & $0.001 \pm 0.001$ & - & $0.002 \pm 0.001$ \\
\hline & Sparisoma axillare (Steindachner. 1878) * & $0.029 \pm 0.014$ & $0.027 \pm 0.012$ & $0.019 \pm 0.008$ & $0.018 \pm 0.007$ \\
\hline & Sparisoma frondosum (Agassiz. 1831) * & $0.003 \pm 0.002$ & - & - & - \\
\hline & Sparisoma sp. & - & - & $0.007 \pm 0.006$ & $0.024 \pm 0.018$ \\
\hline \multirow[t]{3}{*}{ LABRISOMIDAE } & Labrisomus cricota Sazima. Gasparini and Moura. 2002* & - & - & - & $0.001 \pm 0.001$ \\
\hline & Labrisomus nuchipinnis (Quoy and Gaimard. 1824) & $0.013 \pm 0.004$ & $0.007 \pm 0.005$ & $0.014 \pm 0.004$ & $0.038 \pm 0.007$ \\
\hline & Malacoctenus sp. * & $0.016 \pm 0.005$ & $0.017 \pm 0.005$ & $0.028 \pm 0.011$ & $0.043 \pm 0.013$ \\
\hline CHAENOPSIDAE & Emblemariopsis signifera (Ginsburg. 1942) & $0.006 \pm 0.003$ & - & - & - \\
\hline \multirow[t]{4}{*}{ BLENNIIDAE } & Entomacrodus sp. & - & - & $0.002 \pm 0.002$ & $0.001 \pm 0.001$ \\
\hline & Ophioblennius trinitatis Miranda-Ribeiro. 1919 * & $0.004 \pm 0.003$ & $0.043 \pm 0.012$ & $0.167 \pm 0.023$ & $0.159 \pm 0.028$ \\
\hline & Scartella cristata (Linnaeus. 1758) & $0.006 \pm 0.004$ & $0.005 \pm 0.003$ & $0.008 \pm 0.004$ & $0.004 \pm 0.002$ \\
\hline & Parablennius sp. & $0.004 \pm 0.002$ & $0.001 \pm 0.001$ & $0.003 \pm 0.002$ & - \\
\hline \multirow[t]{3}{*}{ GOBIIDAE } & Bathygobius soporator (Valenciennes. 1837) & - & - & - & $0.001 \pm 0.001$ \\
\hline & Coryphopterus sp. & - & $0.001 \pm 0.001$ & - & - \\
\hline & Elacatinus figaro Sazima. Moura and Rosa. 1996 *\# & - & $0.006 \pm 0.006$ & - & - \\
\hline \multirow[t]{3}{*}{ ACANTHURIDAE } & Acanthurus bahianus Castelnau. 1855 & $0.066 \pm 0.037$ & $0.023 \pm 0.009$ & $0.082 \pm 0.019$ & $0.019 \pm 0.008$ \\
\hline & Acanthurus chirurgus (Bloch. 1787) & $0.083 \pm 0.083$ & $0.011 \pm 0.010$ & $0.005 \pm 0.004$ & $0.016 \pm 0.011$ \\
\hline & Acanthurus coeruleus Bloch and Schneider. 1801 & $0.001 \pm 0.001$ & $0.004 \pm 0.003$ & $0.003 \pm 0.002$ & - \\
\hline SPHYRAENIDAE & Sphyraena barracuda (Walbaum. 1792) & - & - & $0.002 \pm 0.002$ & - \\
\hline BOTHIDAE & Bothus ocellatus (Agassiz. 1831) & $0.003 \pm 0.002$ & $0.001 \pm 0.001$ & $0.002 \pm 0.001$ & - \\
\hline MONACANTHIDAE & Cantherhines pullus (Ranzani. 1842) & $0.002 \pm 0.001$ & $0.005 \pm 0.003$ & $0.005 \pm 0.002$ & $0.002 \pm 0.001$ \\
\hline OSTRACIIDAE & Acanthostracion polygonius Poey. 1876 & $0.001 \pm 0.001$ & $0.001 \pm 0.001$ & $0.001 \pm 0.001$ & - \\
\hline \multirow[t]{3}{*}{ TETRAODONTIDAE } & Canthigaster figueiredoi Moura and Castro. $2002 *$ & - & $0.003 \pm 0.002$ & - & - \\
\hline & Sphoeroides greeleyi Gilbert. 1900 & $0.004 \pm 0.003$ & - & - & $0.007 \pm 0.006$ \\
\hline & Sphoeroides spengleri (Bloch. 1785) & $0.011 \pm 0.005$ & $0.025 \pm 0.012$ & $0.017 \pm 0.005$ & $0.023 \pm 0.006$ \\
\hline DIODONTIDAE & Chilomycterus spinosus (Linnaeus. 1758) & - & $0.001 \pm 0.001$ & - & - \\
\hline
\end{tabular}

\footnotetext{
* species endemic to Brazil; \# endangered species.
} 

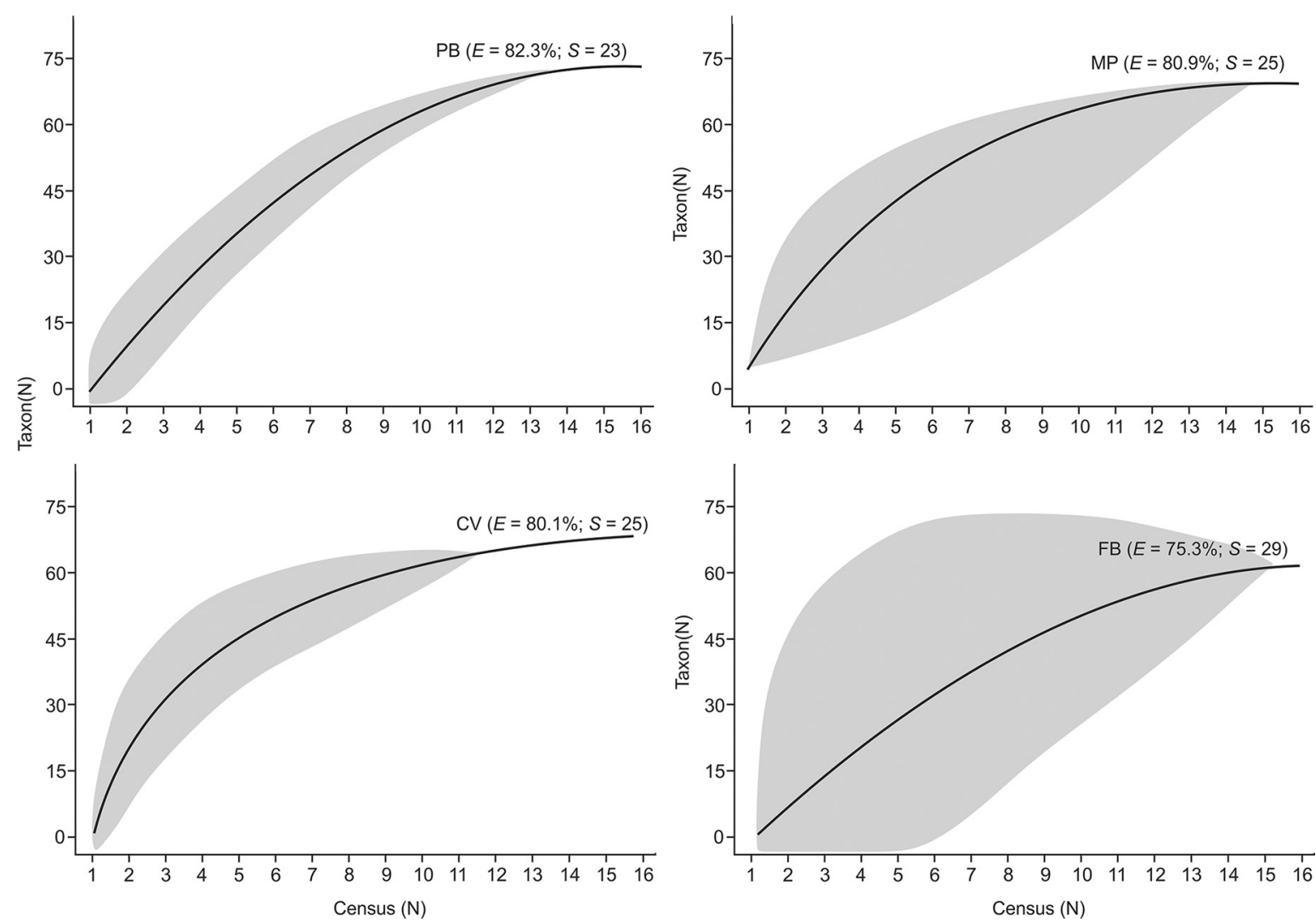

Figure 2. Species accumulation curves obtained by the sixteen underwater visual census in four sampling areas, namely, PB: Porto da Barra; MP: Marco Polo; CV: Corredor da Vitória; FB: Farol da Barra. The shaded area shows the estimated standard deviation. The efficiency of the inventories $(E)$, as the percentage of the censused taxa that potentially occur in the area, and the number of samples $(S)$ necessary to achieve a complete inventory, are also shown.

Vitória (Figure 3). The abundance of the other six species varied moderately amongst sites. The species which were not considered in the graph displayed densities lower than 0.016 fish.m $\mathrm{m}^{-2}$.

The rocky shore fish structure differed significantly between sites (ANOSIM global test: $\mathrm{R}=0.196 ; p=$ $0.0001)$ and significant pairwise differences between sites were recorded (Table 2). The MDS ordination diagrams showed the formation of at least three different groups in the rocky shore fish communities' structure (Figure 4a). Corredor da Vitória had relatively greater dissimilarity than the other sites, although Marco Polo also presented a quite dissimilar pattern.

All sites were dominated by territorial herbivorous fishes ( $\sim 52 \%$ of fishes), followed by mobile invertebrate feeders $(\sim 18 \%)$ and roving herbivores $(\sim 13 \%)$. The other categories were not significantly representative in this study (Figure 5). Only two categories showed significant differences between sites: the territorial herbivores $(p=0.01 ; \mathrm{F}=3.907)$ and the piscivores $(p<0.05 ; \mathrm{F}=3.398)$, with the latter being one of the less representative groups $(0.87 \%)$. Territorial herbivores, mainly represented by the species $S$. fuscus and $O$. trinitatis, showed the highest density values at the Farol da Barra (65.2\%) and Marco Polo (50.7\%) reefs. It is important to highlight that only small piscivores were observed in the census, with the lizard fish, Synodus sp. being the most dominant species.

\section{RELATIONSHIPS BETWEEN FISH COMMUNITIES AND ENVIRONMENTAL VARIABLES}

The ANOVA results showed that rocky shores were homogeneous for the variables: roughness, sand, soft coral, echinoderm cover and rocks; and heterogeneous for the variables: depth, turf algae cover, ascidians, crustose algae, zoanthids, sponges, macroalgae, coral and barnacles (Table 3). Regarding the benthic cover, 

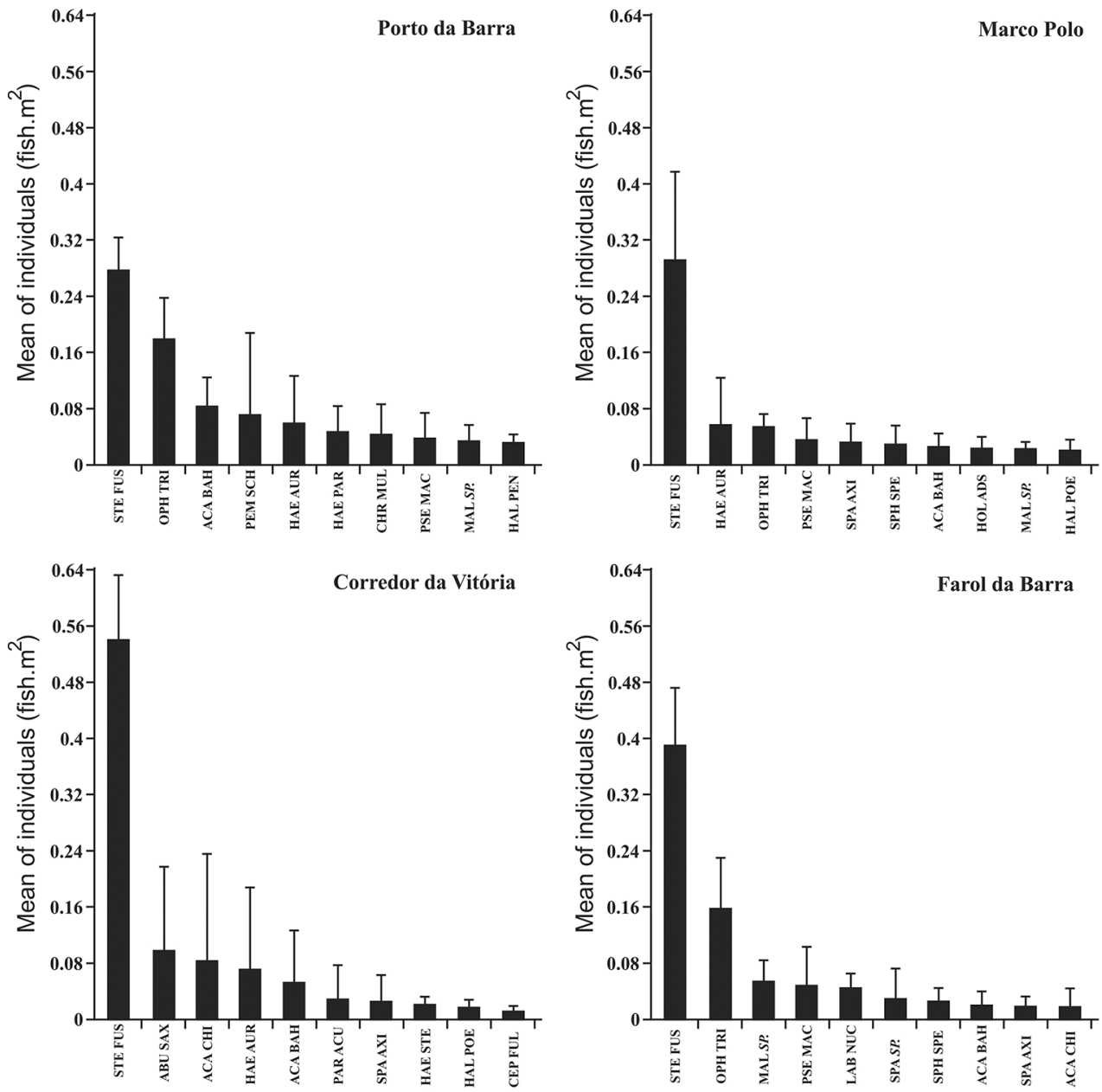

Figure 3. Mean ( \pm Standard deviation - SE) of density of the 10 most abundant species recorded. Stegastes fuscus, Ophioblennius trinitatis, Acanthurus bahianus, Haemulon aurolineatum, Abudefduf saxatilis, Acanthurus chirurgus, Pseudupeneus maculatus, Sparisoma axillare, Pempheris schomburgki and Labrisomus nuchipinnis.

by using a multivariate approach, significant differences were observed between the four rocky shores studied (ANOSIM global test: $\mathrm{R}=0.47 ; p=0.001$ ). The comparison of the pairwise ANOSIM test exhibited an analogous pattern to those found for fish structure, with Corredor da Vitória being the site with the greatest dissimilarity (Table 2). The MDS diagram showed the formation of different groups with Corredor da Vitória being less similar to the other sites, confirming the pairwise ANOSIM results (Figure $4 b$ ).

Turf, macroalgae, roughness, crustose coralline algae and sponge cover were, in decreasing order, the five most important variables influencing fish community distribution, representing $42.5 \%$ of data variability explained on the first axis. The Monte Carlo test showed that the first two axes of the CCA ordination diagram were significant $(p<0.001)$ and accounted for $42.5 \%$ and $26.1 \%$ respectively, of the variance amongst samples, species and variables (Figure 6). The first axis was positively related to roughness and substrate that was dominated by sponges and macroalgae. The second axis was positively associated with roughness, sponges and turf, and negatively associated with macroalgae and crustose coralline algae. There was a clear distinction in species distribution regarding habitat variables. Turf cover clearly influenced most of the species. Stegastes fuscus, P. maculatus and Eucinostomus sp. showed a noticeable positive association with turf cover. On the other hand, the distribution and abundance of A. chirurgus and Cephalopholis fulva were highly influenced by roughness, and $O$. trinitatis distribution was influenced by crustose coralline algae cover. 
Table 2. Fish assemblage and environmental variables results of ANOSIM (Global R) and Pairwise test (Significance level).

\begin{tabular}{lcc}
\hline & Global R & Significance level \\
\hline Fish assemblage & & \\
\hline PB, MP & 0.13 & 0.002 \\
PB, CV & 0.472 & 0.0001 \\
PB, FB & 0.158 & 0.0004 \\
MP, CV & 0.133 & 0.008 \\
MP, FB & 0.109 & 0.009 \\
CV, FB & 0.246 & 0.0001 \\
\hline Benthic cover & & \\
\hline PB, MP & 0.144 & 0.004 \\
PB, CV & 0.845 & 0.0001 \\
PB, FB & 0.187 & 0.005 \\
MP, CV & 0.76 & 0.0001 \\
MP, FB & 0.251 & 0.0004 \\
CV, FB & 0.847 & 0.0001 \\
\hline
\end{tabular}

PB: Porto da Barra; MP: Marco Pólo; CV: Corredor Vitória; FB: Farol da Barra.

\section{DISCUSSION}

\section{COMPOSITION AND STRUCTURE PATTERNS OF}

\section{ROCKY SHORE FISH COMMUNITIES}

The present study is a pioneer research in evaluating the structure of rocky reef fish in northeastern Brazil. Other studies within the same region, which have focused on deep reefs, tidal pools, insular and coastal coral reefs (FEITOZA et al., 2005; GODINHO \& LOTUFO, 2010; MEDEIROS et al., 2010; CHAVES et al., 2010). The MDS diagram in this study presented a slight overlapping of the fish's composition between sites. It is important to note that the sites studied share high densities of some species, such as S. fuscus, O. trinitatis and A. bahianus, which likely explains the MDS result. Yet despite this, the sites are remarkably different regarding their abiotic and biotic characteristics, which may be the principal factor responsible for the differences found in the rocky reef fish community structure. Food availability, directly associated with benthic cover variation, is one of the major factors that may influence these differences. However, other factors should also be taken into account, given that all sites show dissimilarities concerning wave exposure, number of visitors (tourists and fishermen) and boat traffic.

Compared to other rocky shores along the Brazilian coast, in either tropical or subtropical regions, the area in which the present study was carried out is much shallower and narrower (FERREIRA et al., 2001; FLOETER et al., 2007; RANGEL et al., 2007; CHAVES; MONTEIRO, 2009). Despite these differences, this study presented a higher total density of fish $\left(0.75\right.$ ind. $\left.\mathrm{m}^{-2}\right)$ than did the rocky shores studied by Ferreira et al. (2001) $\left(0.37\right.$ ind. $\left.\mathrm{m}^{-2}\right)$ and CHAVES; MONTEIRO (2009) (0.74 ind.m $\left.{ }^{-2}\right)$, yet a lower density than was observed by RANGEL et al. (2007) (1.83 ind. $\mathrm{m}^{-2}$ ) and FLOETER et al. (2007) $\left(1.92\right.$ ind $\left.\mathrm{m}^{-2}\right)$. The presence of a clear variation related to synergic features such as coastal proximity, habitat complexity, anthropogenic activities and fish densities might be strongly related to latitudinal gradients (MORA, 2008; CHAVES; MONTEIRO-NETO, 2009; NUNES et al., 2012). Although subtropical areas may have higher species richness than tropical regions in the South Atlantic, tropical rocky shores can potentially sustain a higher variety of reef fish species than was previously
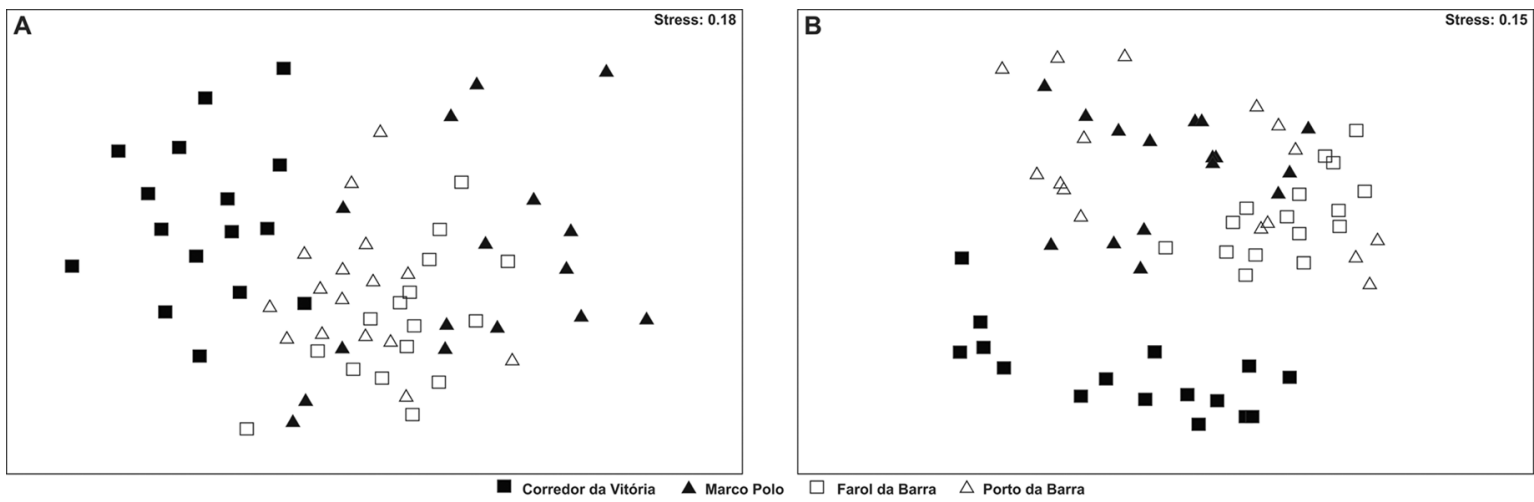

Figure 4. Multidimensional scaling (MDS) based on Bray-Curtis similarity index for: (a) fish communities and; (b) benthic cover compositions. 

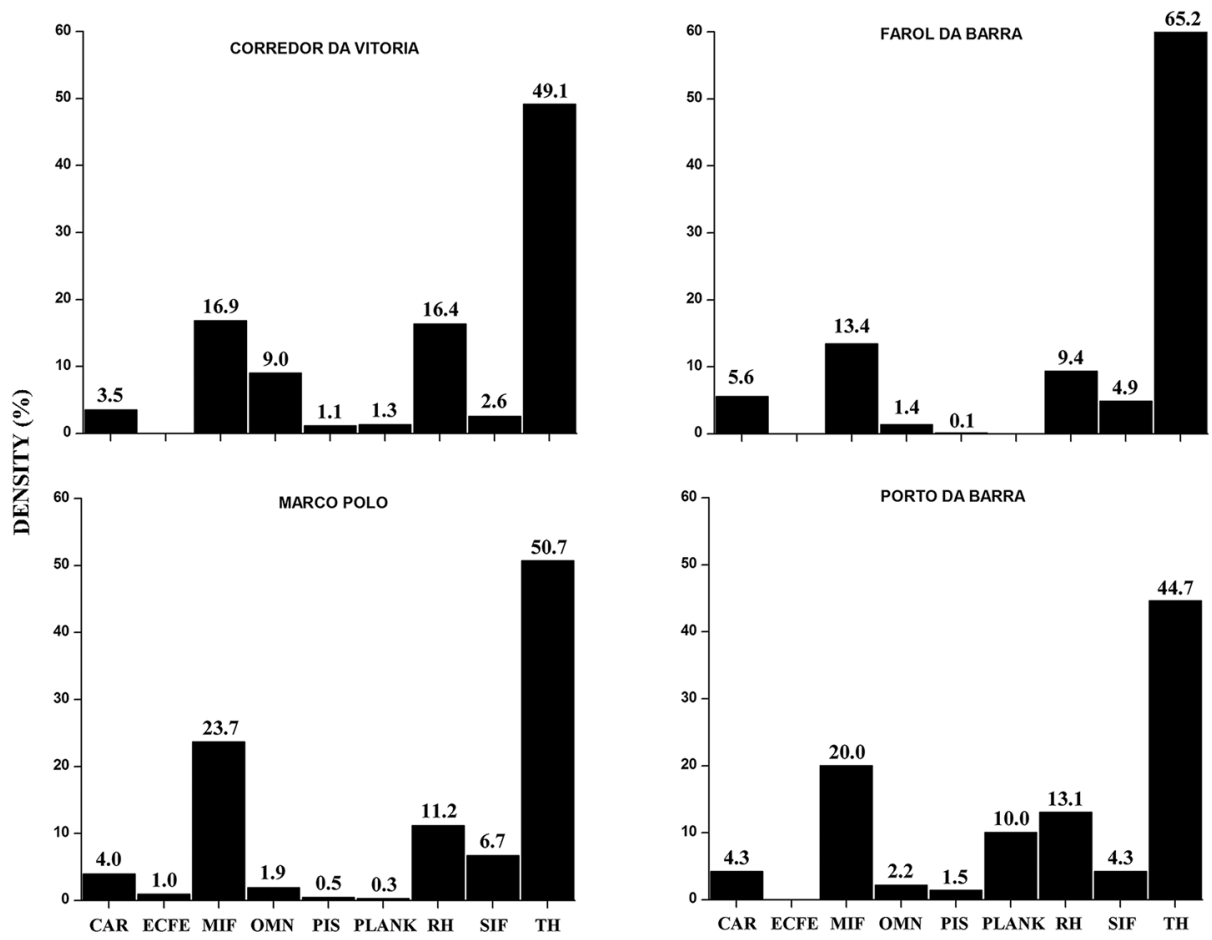

Figure 5. Density (\%) of the trophic composition at each sampled site. CAR: Carnivores; ECTFE: Ectoparasite feeders; MIF: Mobile invertebrate feeders; OMN: Omnivores; PIS: Piscivores; PLK: Planktivores; RH: Roving herbivorous; SIF: Sessile invertebrate feeders; TH: Territorial herbivorous.

Table 3. Environmental variables obtained at sample sites. Mean ( \pm S. E. $)$ and results of ANOVA and SNK tests.

\begin{tabular}{lccccccc}
\hline & PB & MP & CV & FB & F statistic & $p$ & SNK \\
\hline Roughness & $1.34 \pm 0.22$ & $1.34 \pm 0.28$ & $1.29 \pm 0.16$ & $1.21 \pm 0.12$ & 1.49 & 0.22 & - \\
Depth & $2.2 \pm 0.79$ & $3.34 \pm 2$ & $2.57 \pm 0.94$ & $1.7 \pm 0.36$ & 5.41 & 0.002 & MP $>$ CV $\approx$ PB $>$ FB \\
Turf algae & $34.75 \pm 15.68$ & $41.62 \pm 23.75$ & - & $37.21 \pm 14.84$ & 22.7 & 0.000 & MP $>$ PB $\approx \mathrm{FB}>\mathrm{CV}$ \\
Ascidians & $4.15 \pm 4.63$ & $4.56 \pm 6.30$ & $2.06 \pm 2.93$ & - & 4.06 & 0.011 & $\mathrm{~PB} \approx \mathrm{MP}>\mathrm{CV} \approx \mathrm{FB}$ \\
Crustose algae & $7.46 \pm 8.46$ & $3.73 \pm 5.69$ & $5.81 \pm 7.13$ & $12.26 \pm 6.96$ & 4.15 & 0.01 & $\mathrm{FB}>\mathrm{PB} \approx \mathrm{MP} \approx \mathrm{CV}$ \\
Sand & $18.71 \pm 11.47$ & $8.53 \pm 23.08$ & $4.58 \pm 11.92$ & $8.94 \pm 13.43$ & 2.34 & 0.082 & - \\
Soft coral & $8.1 \pm 12.15$ & $5.4 \pm 23.08$ & $5.20 \pm 11.92$ & - & 1.98 & 0.12 & - \\
Equinoderms & $6.43 \pm 11.01$ & $4.56 \pm 11.19$ & $5.83 \pm 11.19$ & $7.88 \pm 6.64$ & 0.6 & 0.61 & - \\
Zoanthids & $9.13 \pm 11.88$ & $6.02 \pm 10.03$ & $10.82 \pm 13.84$ & $23.71 \pm 17.38$ & 5.27 & 0.003 & $\mathrm{FB}>\mathrm{PB} \approx \mathrm{MP} \approx \mathrm{CV}$ \\
Sponges & $1.03 \pm 1.58$ & $1.03 \pm 2.32$ & $4.57 \pm 6.43$ & $9.98 \pm 15.31$ & 4.3 & 0.008 & $\mathrm{CV}>\mathrm{PB} \approx \mathrm{MP} \approx \mathrm{FB}$ \\
Macro algae & $12.47 \pm 13.12$ & $10.82 \pm 4.53$ & $3.74 \pm 8.60$ & $0.61 \pm 1.30$ & 4.3 & 0.008 & $\mathrm{CV}>\mathrm{PB} \approx \mathrm{MP} \approx \mathrm{FB}$ \\
Coral & $3.31 \pm 6.08$ & $7.47 \pm 7.82$ & $3.73 \pm 4.01$ & $1.03 \pm 2.32$ & 3.81 & 0.014 & $\mathrm{MP}>\mathrm{PB} \approx \mathrm{CV} \approx \mathrm{FB}$ \\
Rocks & $2.07 \pm 4.18$ & $7.69 \pm 15.28$ & $9.35 \pm 12.94$ & $1.24 \pm 3.41$ & 2.42 & 0.74 & - \\
Barnacles & - & $5.82 \pm 12.78$ & $0.20 \pm 0.83$ & - & 3.23 & 0.028 & $\mathrm{MP}>\mathrm{PB} \approx \mathrm{CV} \approx \mathrm{FB}$ \\
\hline
\end{tabular}

PB: Porto da Barra; MP: Marco Pólo; CV: Corredor Vitória; FB: Farol da Barra.

thought, and could exceed the number of species found in the southwestern Atlantic's subtropical waters (LUIZJR et al., 2008). However, caution should be exercised when drawing comparisons between other studies and the results obtained here, since the previous studies on rocky shore reefs were performed with different scope, methodologies, sampling effort and temporal and spatial standardization scales. 


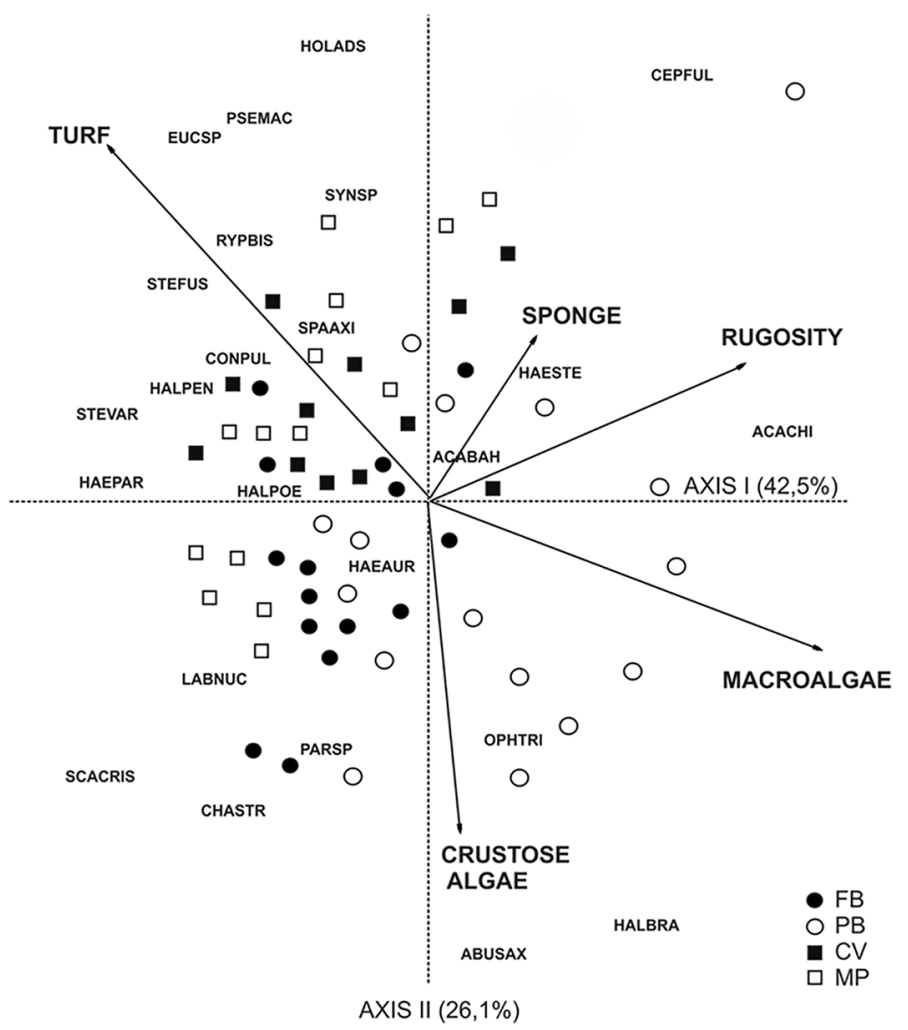

Figure 6. Canonical Correspondence Analysis (CCA) ordination. Species are represented by six letter codes. FB: Farol da Barra; PB: Porto da Barra; CV: Corredor da Vitória; MP: Marco Polo.

The slight spatial variation in species richness found here might be related to the interaction of several factors. Operating both on micro and macro scales, these factors are responsible for differences in species richness, even in small geographical regions (KARLSON; CORNELL, 1998). An important factor already well documented around the world is the intensity and frequency of the effects of natural disturbances on the local richness (CONNEL, 1978; SOUSA, 1979). In general, environments affected by intermediate disturbances potentially hold a higher richness than those affected more severely. This is due to the propensity of intermediate disturbances to slow down the competitive exclusion, occasionally opening new spaces for colonization (CONNEL, 1978). In addition, natural disturbances have induced the fragmentation and presence of different successional stages, increasing the diversity and availability of microhabitats and consequently the number of species (HUSTON, 1985; CHESSON, 1997; JONES; SYMS, 1998). While microhabitat diversity has not been assessed in this study it is believed that the four rocky shores studied present differences in the frequency not only of natural disturbance (e.g. wave exposure) but also of anthropogenic disturbance (NUNES et al., 2013). Additionally, the differences in the number of species present could also be attributed to this. However, to confirm this hypothesis the intensity and frequency of disturbance should be investigated, seeing that the wave exposure in the study area varies seasonally.

Stegastes fuscus (Pomacentridae) was the most abundant species at all the sites, possibly because this species has a preference for sites with low exposure zones, sheltered places (FLOETER et al., 2007), low abundance of competitors (such as large roving herbivorous fish), and high food availability (turf). The Brazilian endemic and cryptic species $O$. trinitatis (Bleniidae) was the second most abundant fish found at two sites, probably for the same reasons as $S$. fuscus. It is possible to think that herbivorous blennies such as $O$. trinitatis evolved to occupy a different niche to damselfishes. Thus they need not compete for food supplies, instead accessing turf algae areas opportunistically. In this way, these two species would be able to share the same area and increase 
both their densities. In the coral reefs of Todos os Santos Bay, Coryphopterus spp. (Gobiidae) are the dominating ones among the cryptic species, a pattern which can also be observed on other southeastern Brazilian rocky shores (FERREIRA et al., 2001; FLOETER et al., 2007; CHAVES; MONTEIRO-NETO, 2009). In addition, the reason why $H$. aurolineatum, $A$. chirurgus, A. bahianus and $A$. saxatiles showed high abundance at some study sites could possibly be because these species are recognized by their characteristic behavior of forming large schools on reef structures, seeking protection, food and reproduction areas (FERREIRA et al., 2004; PEREIRA et al., 2011; FRANCINI-FILHO et al., 2012). The geographical position of the site (the Todos os Santos Bay's mouth), could thus contribute to housing a large quantity of reef fish larvae from reefs in the inner portion. This would provide settlement areas for larvae with rapid development and those with very slow growth rates, unable to reach coral reef areas inside Todos os Santos Bay.

Our results demonstrate that territorial fishes are extremely abundant at all sites and the most representative species in this group by far was Stegastes fuscus ( 78\%). It is well known that territorial fish, especially S. fuscus, are common inhabitants on tropical and shallow reefs where their main food resource (turf) grows better (FERREIRA et al., 1998; CECCARELLI et al., 2001; FLOETER et al., 2007). Turf algae were the most abundant benthic organisms in all the areas studied, which could explain the dominance of territorial fishes. The second most representative trophic group (MIF) includes fishes adapted to a variety of conditions. First of all, the Haemulidae genus showed the highest abundance (40\%), possibly due to their characteristic behavior of forming large and typical schools of up to hundreds of individuals when they are recruits and juveniles (PEREIRA et al., 2011; PEREIRA; FERREIRA, 2013). In addition, some of the sites have an elevated water flow which favors fish species with good swimming skills, such as the members of the Labridae family, which was the second in their contribution to the observed patterns of MIF (FULTON et al., 2001; NUNES et al., 2013). Furthermore, the density of macroinvertebrates inside the S. fuscus territory is higher than that outside it, suggesting that the density of MIFs and S. fuscus might be directly associated (FERREIRA et al., 1998).

The trophic structure results on the Barra rocky shores are different from those reported by RANGEL et al. (2007), where omnivores obtained the highest densities, a pattern that has been observed in other subtropical Brazilian rocky shore studies (FERREIRA et al., 2004; CHAVES; MONTEIRO-NETO, 2009). FERREIRA et al. (2004) state that roving/territorial herbivores and invertebrate feeders are dominant at lower latitudes. However, MOURA and FRANCINI-FILHO (2006) argue that carnivores (fish and/ or invertebrate feeders) were the most abundant group, followed by omnivore, herbivore and planktivore fish on the southern coast of the state of Bahia. The majority of studies cited in this paragraph, as well as our own, were carried out in areas of intense anthropogenic pressure, with the exception of MOURA and FRANCINI-FILHO (2006), whose study was performed in a Marine Protected Area (the Abrolhos bank). Anthropogenic disturbances are known to have a major impact upon the food web, interfering throughout the whole community structure. Regarding trophic structure, it is likely that our results were influenced by abiotic and biotic characteristics, as the sites are relatively more sheltered and have warmer waters than other rocky shore regions. To illustrate this, the low abundance of the planktivorous fish (Chromis spp.) is likely due to the decrease of wave exposure. It is commonly understood that planktivorous fish prefer turbulent waters with a higher availability of plankton (FLOETER et al., 2007).

RELATIONSHIP BETWEEN FISH COMMUNITIES AND ENVIRONMENTAL VARIABLES

Subtidal benthic communities on rocky shores are likely to have a less diverse algae community in the Todos os Santos bay (MARINS et al., 2008). In the present study, turf algae cover was the dominant benthic organism by far. This same benthic structure pattern had been found by former studies on Brazilian coral reefs (FRANCINI-FILHO; MOURA， 2008; FRANCINIFILHO et al., 2013) and rocky shores (FERREIRA et al., 2001; FLOETER et al., 2007). Turf algae is recognized as a conspicuous organism in shallow areas due to its dependence upon sunlight (FERREIRA et al., 1998). However, many studies have shown that they can be detrimental to the vitality of coral reefs (VERMEIJ et al., 2010; BAROTT et al., 2012). Nevertheless, these effects remain controversial as they may vary depending on turf and corals species composition (BIRRELL et al., 2005), with some coral species appearing to be unaffected by them (JOMPA; MCCOOK, 2003). Furthermore, turf algae can have positive consequences upon communities, serving as a food resource for many organisms (FERREIRA; GONÇALVES, 2006; FRANCINI-FILHO et al., 2010). 
In the CCA results the majority of the observed species were positively associated with turf algae. This association with some species such as S. fuscus and Sparisoma axillare is to be expected, given that turf algae is their preferred food source (FLOETER et al., 2007; FRANCINI-FILHO et al., 2010). Yet surprisingly, the same pattern also occurred with other species, such as P. maculatus and Eucinostomus sp. These species are typical fossorial feeders, foraging mostly over sand (CARVALHO-FILHO, 1999). A potential explanation for this pattern is the fact that large areas with an interface of sand and turf substrate are easily seen across the study sites. Another possible explanation is that in association with turf algae, there are many other components, such as invertebrates and microorganisms composition, detritus and sediment (CHOAT, 1991), which could also explain their association with invertebrate feeders (e.g. Haemulon parrae, Halichoeres poey and $H$. penrosei).

Another important positive relationship observed was between Acanthurus spp. and roughness. Acanthurus bahianus and A. chirurgus are common inhabitants of complex habitats, such as those with fire corals (CONI et al., 2013). Even though the Barra rocky shore did not have a great amount of fire coral, its roughness can provide sufficient protection for a variety of fishes, such as the carnivorous $C$. fulva. The higher habitat complexity can increase the rates of predation success, due to the amount of prey availability (HIXON, 1993). Ophioblennius trinitatis showed a high association with crustose coralline cover mainly at Porto da Barra and Farol da Barra, sites with the highest rates of crustose algae cover (see Table 3 ). It is likely that this fish scrapes algae microfilm that overgrows crustose coralline algae.

The present results show the high variability between fish community structure and habitat complexity relationship found in reef systems. SALE (1991) observed that structuring habitat factors can differ between locations and that fish communities are not predictable. MEDEIROS et al. (2010), studying Brazilian coastal reefs, found that depth and substrate composition, particularly macroalgae cover, were positive determinants for the fish community structure. According to these authors, the presence of macroalgae is one of the components that enhance habitat complexity, and thus provide shelter for small and/or juvenile individuals of many reef fish species. However, for the four rock shores sampled, food availability (e.g., turf algae) might be the principle factor responsible for the fish community structure.

\section{CONSERVATION REMARKS}

The rocky shores studied are under intense human pressure, primarily due to pollution, fishing and/or tourism activities (authors, per. obs.). Clear waters, shallow depths and free access encourage intense fishing practices in the region, potentially making it the area most frequented by spear fishermen on the coast of the state of Bahia (NUNES et al., 2012). Currently, reports of marine ornamental fish in these areas are becoming rare, mainly due to the intense capture of these species since the 1970s (SAMPAIO; NOTTHINHAM, 2008). To illustrate this, during the study period, there were only a few reports of the neon goby Elacatinus figaro. Due to its bright color, this species is extremely attractive to the ornamental industry, and it is currently classified as endangered by the Brazilian Red List (IUCN) (MACHADO et al., 2008; ROSA; LIMA, 2008; SAMPAIO; NOTTHINHAM, 2008).

Until the mid-1980s, the dog snappers Lutjanus jocu, the great barracuda Sphyraena barracuda, the stingrays Dasyatis spp. and the Atlantic goliath grouper Ephinephelus itajara were observed regularly (authors, per. obs.). Currently, just few individuals and/or only juveniles can be sporadically spotted in the area. Another evident reduction is in the biomass of roving herbivorous fishes, such as Scarus trispinosus, which is known to be an important functional group keeping the algal and coral populations under control (BELLWOOD et al., 2004; HUGHES, 1994, 2007, 2010; MCCOOK, 1999; SZMANT, 2002; NORSTRÖM et al., 2009). The extirpation of these fish from the reef systems can lead to severe negative consequences (DUFFY, 2003). It is widely accepted that the predator-prey relationship is a key to the ecological balance of the communities, and therefore that the removal of predators from reef environments could affect the entire reef community structure (FLOETER et al., 2007; FRANCINI-FILHO et al., 2010). Undoubtedly, the intense fishing exploitation on the Barra rocky shores has reduced the abundance of competitors and predators (such as roving herbivorous and large carnivorous fish, both fisheries target species), and consequently, increased the abundance of territorial herbivorous and invertebrate feeders (non-fisheries species). The degree of impact this has had upon the rocky shores remains unknown, given that this region has been exploited for approximately five centuries.

Although information regarding the community structure of Brazilian reef fishes is relatively widespread, the majority of the studies undertaken were performed on 
tropical coral reefs and rocky shores in temperate zones (EBELING; HIXON, 1991). In comparison, there are few studies involving the associations among fishes on tropical rocky shores (CONI et al., 2007, 2010; NUNES et al., 2007a, 2007b; MEDEIROS et al., 2009a; 2009b; MAIA-NOGUEIRA et al., 2010; MEDEIROS et al., 2011). Thus this region still remains largely unknown. The purpose of this study was to take the initial step to contribute to broadening future studies on shallow coral reefs, shipwrecks and deep reefs near the rocky shores studied here. Ultimately, the northeastern rocky shores deserve special attention, since they are located at the mouth of the Todos os Santos Bay, an area accorded top priority for biological conservation (PASSOS et al., 2002). In addition, these rocky shores lie in a strategic position connecting the internal and external reef environments of the bay and contributing directly to the continuous interchange of species.

\section{ACKNOWLEDGEMENTS}

We thank Laís Chaves and Rodrigo Maia-Nogueira for their valuable help during the fieldwork and for kindly reviewing this manuscript. This study received no specific grant from any funding agency, commercial or non-profit sectors. FB was supported by a fellowship PQ-CNPq (№ 302642/2008-0).

\section{REFERENCES}

BAROTT, K. L.; WILLIAMS, G. J.; VERMEIJ, M. J.; HARRIS, J.; SMITH, J. E.; ROHWER, F. L.; SANDIN, S. A. Natural history of coral-algae competition across a gradient of human activity in the Line Islands. Mar. Ecol. Prog. Ser., v. 460, p. 1-12, 2012.

BELLWOOD, D. R.; HUGHES, T. P.; FOLKE, C.; NYSTRÖM, M. Confronting the coral reef crisis. Nature, v. 429, p. 827833, 2004.

BIRRELL, C. L.; MCCOOK, L. J.; WILLIS, B. L. Effects of algal turfs and sediment on coral settlement. Mar. Pollut. Bull., v. 51, n. 1/4, p. 408-414, 2005.

BROCK, V. E. A preliminary report on a method of estimating reef fish populations. J. Wild. Manag., v. 18, n. 3, p. 297-308, 1954.

BRUCE, T.; MEIRELLES, P. M.; GARCIA, G.; PARANHOS, R.; REZENDE, C. E.; DE MOURA, R. L.; FRANCINI FILHO, R. B.; CONI, E. O. C.; VASCONCELOS, A. T.; AMADO FILHO, G.; HATAY, M.; SCHMIEDER, R.; EDWARDS, R.; DINSDALE, E.; THOMPSON, F. L. Abrolhos bank reef health evaluated by means of water quality, microbial diversity, benthic cover, and fish biomass data. PLoS ONE, v. 7, n. 6, p. e36687, 2012.
CARVALHO-FILHO, A. Peixes: costa brasileira. 3.ed. São Paulo: Melro, 1999. 320 p.

CASTRO, C. B.; PIRES, D. O. Brazilian coral reefs: what we already know and what is still missing. Bull. Mar. Sci., v. 69, n. 2, p. 357-371, 2001.

CECCARELLI, D. M.; JONES G. P.; MCCOOK, L. J. Territorial damselfishes as determinants of the structure of benthic communities on coral reefs. Oceanogr. Mar. Biol. Ann. Rev., v. 39, p. 355-389, 2001.

CHAVES, L. C. T.; MONTEIRO-NETO, C. Comparative analysis of rocky reef fish community structure in coastal islands of south-eastern Brazil. J. Mar. Biol. Assoc. U. K., v. 89, n. 3, p. 609-619, 2009.

CHAVES, L. C. T.; NUNES, J. A. C. C.; SAMPAIO, C. L. S. Shallow reef fish communities of south Bahia coast, Brazil. Braz. J. Oceanogr., v. 58, n. spe 4, p. 33-46, 2010.

CHESSON, P. L. Diversity maintenance by integration of mechanisms over various scales. Proc. 8th Int. Coral Reef Symp., v. 1, p. 405-410, 1997.

CHOAT, J. H. The biology of herbivorous fishes on coral reefs. In: SALE, P. F. (Ed.). The Ecology of Fishes on Coral Reefs. San Diego: Academic press, 1991. p. 120-155.

CIRANO, M.; LESSA, G. C. Oceanographic characteristics of Baía de Todos os Santos Bay, Brasil. Braz. J. Geophys., v. 25, n. 4, p. 363-387, 2007.

CONI, E. O.; NUNES, J. A. C. C.; SAMPAIO, C. L. S. Halichoeres penrosei (Labridae), a sporadic cleaner wrasse. J. Mar. Biol. Assoc. U. K., v. 1, p. 1-2, 2007.

CONI, E. O. C.; NUNES, J. A. C. C.; FERREIRA, C. M.; MAIANOGUEIRA, R.; MEDEIROS, D. V.; SAMPAIO, C. L. S. The Spanish hogfish Bodianus rufus (Labridae) acting as cleaner of nocturnal fish in the north-east of Brazil. Mar. Biod. Rec., v. 3, n. e23, p. 2, 2010.

CONI, E. O. C.; FERREIRA, C. M.; MOURA, R. L.; MEIRELLES, P. M.; KAUFMAN, L.; FRANCINI-FILHO, R. B. An evaluation of the use of branching fire-corals (Millepora spp.) as refuge by reef fish in the Abrolhos Bank, eastern Brazil. Environ. Biol. Fish., v. 96, n. 1, p. 45-55, 2013.

CRAIG, M. T.; HASTINGS, P. A. A molecular phylogeny of the groupers of the subfamily Epinephelinae (Serranidae) with a revised classification of the Epinephelini. Ichthyol. Res., v. 54, n. 1, p. 1-17, 2007.

CROSBY, M. P.; REESE, E. S. A Manual for monitoring coral reefs with indicator species: Butterflyfishes as Indicators of Change on Indo-Pacific Reefs. Maryland: Silver Spring, 1996. $47 \mathrm{p}$.

DE RAEDEMAECKER, F.; MILIOU, A.; PERKINS, R. Fish community structure on littoral rocky shores in the Eastern Aegean Sea: Effects of exposure and substratum. Estuar. Coast. Shelf Sci., v. 90, n. 1, p. 35-44, 2010.

DUFFY, J. E. Biodiversity loss, trophic skew and ecosystem functioning. Ecol. Lett., v. 6, n. 8, p. 680-687, 2003.

EBELING, A. W.; HIXON, M. A. Tropical and Temperate reef fishes: Comparison of Community Structures. In: SALE, P. F. S. (Ed.). The Ecology of Fishes on Coral Reefs. San Diego: Academic press, 1991. p. 509-563.

FEITOZA, B. M.; ROSA, E. S.; ROCHA, L. A. Ecology and zoogeography of deep reef fishes in northeastern Brazil. Bull. Mar. Sci., v. 76, n. 3, p. 725-742, 2005. 
FERREIRA, C. E. L.; GONÇALVES, J. E. A.; COUTINHO, R.; PERET, A. C. Herbivory by the Dusky Damselfish, Stegastes fuscus (Cuvier, 1830) in a tropical rocky shore: effects on the benthic community. J. Exp. Mar. Biol. Ecol., v. 229, n. 2, p. 241-264, 1998.

FERREIRA, C. E. L.; GONÇALVES, J. E. A.; COUTINHO, R. Fish community structure and habitat complexity in a tropical rocky shore. Environ. Biol. Fish., v. 61, n. 4, p. 353-369, 2001.

FERREIRA, C. E. L.; GONÇALVES, J. E. A. Community structure and diet of roving herbivorous fishes in the Abrolhos Archipelago, south-western Atlantic. J. Fish Biol., v. 69, n. 4, p. 1533-1551, 2006.

FERREIRA, C. E. L.; FLOETER, S. R.; GASPARINI, J. L.; JOYEUX, J. C.; FERREIRA, B. P. Trophic structure patterns of Brazilian reef fishes: a latitudinal comparison. J. Biogeogr., v. 31, n. 7, p. 1093-1106, 2004.

FLOETER, S. R. F.; GASPARINI, J. L.G. The southwestern Atlantic reef fish fauna: composition and zoogeographic patterns. J. Fish Biol., v. 56, n. 5, p. 1099-1114, 2000.

FLOETER, S. R.; HALPERN, B. S.; FERREIRA, C. E. L. Effects of fishing and protection on Brazilian reef fishes. Biol. Conserv., v. 128, n. 3, p. 391-402, 2006.

FLOETER, S. R.; KROHLING, W.; GASPARINI, J. L.; FERREIRA, C. E. L.; ZALMON, I. Reef fish community structure on coastal islands of the southeastern Brazil: the influence of exposure and cover. Environ. Biol. Fish., v. 78, n. 2, p. 147-160, 2007.

FRANCINI-FILHO, R. B.; MOURA, R. L.; THOMPSON, F. L.; REIS, R. M.; KAUFMAN, L.; KIKUCHI, R. K. P.; LEÃO, Z. M. A. N. Diseases leading to accelerated decline of reef corals in the largest South Atlantic reef complex (Abrolhos Bank, eastern Brazil). Mar. Pollut. Bull., v. 56, n. 5, p. 1008$1014,2008$.

FRANCINI-FILHO, R. B.; MOURA, R. L. Dynamics of fish assemblages on coral reefs subjected to different management regimes in the Abrolhos Bank, eastern Brazil. Aquat. Conserv., v. 18, n. 7, p. 1166-1179, 2008.

FRANCINI-FILHO, R. B.; FERREIRA, C. M.; CONI, E. O. C.; MOURA, R. L.; KAUFMAN, L. Foraging activity of roving herbivorous reef fish (Acanthuridae and Scaridae) in eastern Brazil: influence of resource availability and interference competition. J. Mar. Biol. Assoc. U. K., v. 90, n. 3, p. 481492, 2010.

FRANCINI-FILHO, R. B.; CONI, E. O. C.; FERREIRA, C. M.; ALVES, A. C.; AMADO-FILHO, G. Group nest clearing behavior by the sergeant major Abudefduf saxatilis (Pisces: Pomacentridae). Bull. Mar. Sci., v. 88, n. 2, p. 195-196, 2012.

FRANCINI-FILHO, R. B.; CONI, E. O. C.; MEIRELLES, P. M.; AMADO-FILHO, G. M.; THOMPSON, F. L.; PEREIRAFILHO, G. H.; BASTOS, A. C.; ABRANTES, D. P.; FERREIRA, C. M.; GIBRAN, F. Z.; GÜTH, A. Z.; SUMIDA, P. Y. G.; OLIVEIRA, N. L.; KAUFMAN, L.; MINTEVERA, C. V.; MOURA, R. L. Dynamics of coral reef benthic assemblages of the Abrolhos Bank, eastern Brazil: inferences on natural and anthropogenic drivers. PLoS ONE, v. 8, n. 1, p. e54260, 2013.

FROESE, R.; PAULY, P. FishBase. World Wide Web electronic publication. Available at: <www.fishbase.org $>$. Accessed: 02/2014.
FULTON, C. J., BELLWOOD, D. R.; WAINWRIGHT, P. C. The relationship between swimming ability and habitat use in wrasses (Labridae). Mar. Biol., v. 139, n. 1, p. 25-33, 2001.

GODINHO, W. O.; LOTUFO, T. M. C. Local v. microhabitat influences on the fish fauna of tidal pools in northeast Brazil. J. Fish Biol., v. 76, n. 3, p. 487-501, 2010.

GOTELLI, N.; COLWELL, R. K. Quantifying biodiversity: procedures and pitfalls in the measurement and comparison of species richness. Ecol. Lett., v. 4, n. 4, p. 379-391, 2001.

GUST, N. Scarid biomass on the northern Great Barrier Reef: the influence of exposure, depth and substrata. Environ. Biol. Fish., v. 64, n. 4, p. 353-366, 2002.

HIXON, M. Predation, prey refuges, and the structure of coral-reef fish assemblages. Ecol. Monogr., v. 63, n. 1, p. 77-101, 1993.

HIXON, M. A.; BEETS, J. P. Predation, prey refuges and the structure of coral-reef fish assemblages. Ecol. Monogr., v. 63, n. 1, p. 77-101, 1993.

HOSTIM-SILVA, M.; ANDRADE, A. B.; MACHADO, L. F.; GERHARDINGER, L. C.; DAROS, F. A.; BARREIROS, J. P.; GODOY, E. A. S. Peixes de costão rochoso de Santa Catarina: Ilha do Arvoredo. Itajaí: Universidade do Vale do Itajaí, 2006. $134 \mathrm{p}$.

HUGHES, T. P. Catastrophes, phase shifts, and large-scale degradation of a Caribbean coral reef. Science, v. 265, n. 5178, p. 1547-1551, 1994.

HUGHES, T. P.; RODRIGUES, M. J.; BELLWOOD, D. R.; CECCARELLI, D.; HOEGH-GULDBERG, O.; MCCOOK, L.; MOLTSCHANIWSKYJ, N.; PRATCHETT, M. S.; STENECK, R. S.; WILLIS, B. Phase shifts, herbivory and the resilience of coral reefs to climate change. Curr. Biol., v. 17, n. 4, p. 360-365, 2007.

HUGHES, T. P.; NICHOLAS, A. J.; GRAHAM, J. B. C.; JACKSON, P. J. M.; ROBERT, S. S. Rising to the challenge of sustaining coral reef resilience. Trends Ecol. Evol., v. 25, n. 11, p. 633-641, 2010.

HUSTON, M. A. Patterns of species diversity on coral reefs. Annu. Rev. Ecol. Syst., v. 16, p. 149-177, 1985.

JOMPA, J.; MCCOOK, L. J. Contrasting effects of turf algae on corals: massive Porites spp. are unaffected by mixed-species turfs, but are killed by the red alga Anotrichium tenue. Mar. Ecol. Prog. Ser., v. 258, p. 79-86, 2003.

JONES, G. P. Ecology of rocky reef fish of north-eastern New Zealand: a review. N. Z. J. Mar. Freshwater Res., v 22, n. 3, p. 445-462, 1988.

JONES, G. P.; SYMS, C. Disturbance, habitat structure and the ecology of fishes on coral reefs. Aust. J. Ecol., v. 23, n. 3, p. 287-297, 1998.

KARLSON, R. H.; CORNELL, H. V. Scale-dependent variation in local vs. regional effects on coral species richness. Ecol. Monogr., v. 68, n. 2, p. 259-274, 1998.

LEÃO, Z. M. A. N.; KIKUCHI, R. K. P.; TESTA, V. Corals and Coral Reefs of Brazil. In: CORTÉS, J. (Ed.). Latin American Coral Reefs. Amsterdam: Elsevier, 2003. p. 9-52.

LeSsA, G. C.; BITTENCOURT, A. C. S. P.; BRICHTA, A.; DOMINGUEZ, J. M. L. A reevaluation of the late quaternary sedimentation in Todos os Santos Bay (BA), Brazil. An. Acad. Bras. Cienc., v. 72, n. 4, p. 573-590, 2000. 
LUCKHURST, B. E.; LUCKHURST, K. Analysis of influence of substrate variables on coral reef fish communities. Mar. Biol., v. 49, p. 317-324, 1978.

LUIZ-JR, O. J; CARVALHO-FILHO, A.; FERREIRA, C. E. L.; FLOETER, S. R.; GASPARINI, J. L.; SAZIMA, I. The reef fish assemblage of the Lage dos Santos Marine State Park, Southwestern Atlantic: annotated checklist with comments on abundance, distribution, trophic structure, symbiotic association, and conservation. Zootaxa, v. 1807, p. 1-25, 2008.

MACHADO, A. B. M.; DRUMMOND, G. M.; PAGLIA, A. P. (Eds.). Livro Vermelho da Fauna Brasileira Ameaçada de Extinção. Brasília: Ministério do Meio Ambiente, 2008. p. 188-189.

MAIA-NOGUEIRA, R.; MEDEIROS, D. V.; NUNES, J. A. C. C.; SAMPAIO, C. L. S. Banded butterflyfish Chaetodon striatus (Chaetodontidae) cleaning the green turtle, Chelonia mydas (Cheloniidae). Mar. Biod. Rec., v. 3, p. e116, 2010.

MARINS, B. V.; BRASILEIRO, P. S.; BARRETO, M. B. B.; NUNES, J. M. C.; YONESHIGUE-VALENTIM, Y.; AMADO FILHO, G. M. Subtidal benthic marine algae of the Todos os Santos Bay, Bahia State, Brazil. Oecol. Bras., v. 12, n. 2, p. 229-242, 2008.

MCCOOK, L. J. Macroalgae, nutrients and phase shifts on coral reefs: scientific issues and management consequences for the Great Barrier Reef. Coral Reefs, v. 18, n. 4, p. 357-367, 1999.

MCCUNE, B.; GRACE, J. B. Analysis of ecological communities. Gleneden Beach: MjM Software Design. 2002. p. 190-191.

MEDEIROS, D. V.; NUNES, J. A. C. C.; SAMPAIO, C. L. S. A mutton hamlet Alphestes afer (Bloch, 1793) reproductive event in northeast Brazil. Panam. J. Aquat. Sci., v. 4, n. 2, p. 212-215, 2009a.

MEDEIROS, D. V.; NUNES, J. A. C. C.; REIS-FILHO, J. A.; SAMPAIO, C. L. S. Yellowline arrow crab Stenorhynchus seticornis (Brachyura: Majidae) acting as a cleaner of reef fish, eastern Brazil. Mar. Biodivers. Rec., v. 4, n. e68, 2009b.

MEDEIROS, P. R.; GREMPEL, R. G.; SOUZA, A. T.; ILARRI, M. I.; ROSA, R. S. Non-random reef use by fishes at two dominant zones in a tropical, algal-dominated coastal reef. Environ. Biol. Fish., v. 87, n. 3, p. 237-246, 2010.

MEDEIROS, P. R.; ROSA, R. S.; FRANCINI-FILHO, R. B. Dynamics of fish assemblages on a continuous rocky reef and adjacent unconsolidated habitats at Fernando de Noronha Archipelago, tropical western Atlantic. Neotrop. Ichtyol., v. 9, n. 4, p. 869-879, 2011.

MOBERG, F.; FOLKE, C. Ecological goods and services of coral reef ecosystems. Ecol. Econ., v. 29, n. 2, p. 215-233, 1999.

MORA, C. A clear human footprint in the coral reefs of the Caribbean. Proc. Roy. Soc. Lond. B., v. 275, n. 1636, p. 767773, 2008.

MOURA, R. L. Brazilian reefs as priority areas for biodiversity conservation in the Atlantic Ocean. Proc. 9th Int. Coral Reef Symp., v. 9, n. 2, p. 917-920, 2003.

MOURA, R. L.; FRANCINI-FILHO, R. B. Reef and shore fishes of the Abrolhos Region, Brazil. In: DUTRA, G. F.; ALLEN, G. R.; WERNER, T.; MCKENNA, A. S. (Eds.). A Rapid Marine Biodiversity Assessment of the Abrolhos Bank, Bahia, Brazil. Washington: Conservation International, 2006. p. 40-55.
NELSON, J. S. Fishes of the World. 4th ed. New York: Wiley, 2006. $601 \mathrm{p}$.

NORSTRÖM, A.V.; NYSTRÖM, M.; LOKRANTZ, J.; FOLKE, C. Alternative states on coral reefs: beyond coral - macroalgal phase shifts. Mar. Ecol. Prog. Ser., v. 376, p. 295-306, 2009.

NUNES, J. A. C. C.; CHAVES, L. T.; MAIA-NOGUEIRA, R.; SAMPAIO, C. L. S. Association between juvenile reef fish and the Caribbean reef squid Sepioteuthis sepioidea on northeastern Brazilian coastal reefs. J. Mar. Biol. Assoc. U. K., v. 87, n. 3, p. 761-762, 2007a.

NUNES, J. A. C. C.; MAIA-NOGUEIRA, R.; SAMPAIO, C. L. S. "Friendly behavior" between two species of Myrichthys in Brazilian waters. Coral Reefs, v. 26, p. 199, 2007 b.

NUNES, J. A. C. C.; MEDEIROS, D. V.; REIS-FILHO, J. A.; SAMPAIO, C. L. S.; BARROS, F. Reef fishes captured by recreational spearfishing on reefs of Bahia state, northeast Brazil. Biota. Neotrop., v. 12, n. 1, p. 179-185, 2012.

NUNES, J. A. C. C.; SAMPAIO, C. L. S.; BARROS, F. How wave exposure, group size and habitat complexity influence foraging and population densities in fishes of the genus Halichoeres (Perciformes: Labridae) on tropical rocky shores. Mar. Biol., v. 160, n. 9, p. 2383-2394, 2013.

OHLHORST, S. L.; LIDDELL, W. D.; TAYLOR, R. J.; TAYLOR, J. M. Evaluation of reef census techniques. Proc. 6th Int. Coral Reef Symp., v. 2, p. 319-324. 1988.

OKSANEN, J.; BLANCHET, F. G.; KINDT, R.; LEGENDRE, R.; MINCHIN, P. R.; O'HARA R. B.; SIMPSON, G. L.; SOLYMOS, P.; STEVENS, M. H. H.; WAGNER, H. Vegan: community ecology package, R Package Version 2.0-10, 2013.

PANDOLFI, J. M.; BRADBURY, R. H.; SALA, E.; HUGHES, T. P.; BJORNDAL, K. A.; COOKE, R. G.; MCARDLE, D.; MCCLENACHAN, L.; NEWMAN, M. J. H.; PAREDES, G.; WARNER, R. R.; JACKSON, J. B. C. Global trajectories of the long-term decline of coral reef ecosystems. Science, v. 301, n. 5635, p. 955-958, 2003.

PASSOS, C. M. O.; PEREIRA, R. M.; MONTEIRO, M. M. Avaliação e ações prioritárias para a conservação da biodiversidade das zonas costeiras marinhas. Brasília: Ministério do Meio Ambiente, 2002.

PAULY, D.; CHRISTENSEN, V. Primary production required to sustain global fisheries. Nature, v. 374, p. 255-257, 1995.

PEREIRA, P. H. C.; FEITOSA, J. L. L.; FERREIRA, B. P. Mixedspecies schooling behavior and protective mimicry involving coral reef fish from the genus Haemulon (Haemulidae). Neotrop. Ichthyol., v. 9, n. 4, p. 741-746, 2011.

PEREIRA P. H. C.; FERREIRA, B. P. Effects of life phase and schooling patterns on the foraging behaviour of coral-reef fishes from the genus Haemulon. J. Fish. Biol., v. 82, n. 4, p. 1226-1238, 2013.

PEREIRA-FILHO, G. H.; AMADO-FILHO, G. M.; GUIMARÃES, S. M. P. B.; MOURA, R. L.; SUMIDA, P. Y. G.; ABRANTES, D. P.; BAHIA, R. G.; GÜTH, A. Z.; JORGE, R. R.; FRANCINI FILHO, R. B. Reef fish and benthic assemblages of the Trindade and Martin Vaz Island group, southwestern Atlantic. Braz. J. Oceanogr., v. 59, n. 3, p. 201-212, 2011.

RANDALL, J. E. Food habits of reef fishes of the West Indies. Stud. Trop. Oceanogr., v. 5, p. 665-847, 1967. 
RANGEL, C. A.; CHAVES, L. C. T.; MONTEIRO-NETO, C. Baseline assessment of the reef fish assemblage from Cagarras archipelago, Rio de Janeiro, southeastern Brazil. Braz. J. Oceanogr., v. 55, n. 1, p. 7-17, 2007.

REAKA-KUDLA, M. L. The global biodiversity of coral reefs: a comparison with rain forests. In: REAKA-KUDLA, M. L.; WILSON, D. E.; WILSON, E. O. (Eds.). Biodiversity II. Washington: Joseph Henry Press, 1997. pp 83-108.

REIS-FILHO, J. A.; BARROS, F.; NUNES, J. A. C. C.; FERREIRA, A. Estuarine ichthyofauna of the Paraguaçu River, Todos os Santos Bay, Bahia, Brazil. Biota Neotrop., v. 10, n. 4, p. 301-311, 2010.

REIS-FILHO, J. A.; BARROS, F; NUNES, J. A. C. C.; SAMPAIO, C. L. S.; SOUZA, G. B. G. Moon and tide effects on fish capture in a tropical tidal flat. J. Mar. Biol. Assoc. U. K., v. 1, p. 1-9, 2011.

ROGERS, C. S.; GARRISON, G.; GROBER, R.; HILLIS, Z. M.; FRANKE, M. A. Coral Reef Monitoring Manual for the Caribbean and Western Atlantic. St John: National Park Service, 1994. 114 p.

ROSA, R. S.; LIMA, F. C. T. Peixes. In: BARBOSA, A.; MACHADO, M.; DRUMMOND, G. M.; PAGLIA, A. P. (Eds.). Livro vermelho da fauna brasileira ameaçada de extinção. Belo Horizonte: Fundação Biodiversitas, 2008. p. 8-285.

RUSS, G. R. Coral reef fisheries: effects and yields. In: SALE, P. F. (Ed.). The Ecology of Fishes on Coral Reefs. San Diego: Academic press, 1991. p. 601-635.

RUSS, G. R.; ALCALA, A. C. Management histories of Sumilon and Apo Marine Reserves, Philippines, and their influence on national marine resource policy. Coral Reefs, v. 18, n. 4, p. 307-319, 1999.
SALE, P. F. The ecology of fishes on coral reefs. London: Academic Press, 1991. 754 p.

SAMPAIO, C. L. S.; NOTTINGHAM, M. C. Guia para identificação de peixes ornamentais. Brasília: Ibama, 2008. $205 \mathrm{p}$.

SOUSA, W. P. Disturbance in marine intertidal boulder fields: the noneequilibrium maintenance of species diversity. Ecology, v. 60 , n. 6, p. 1225-1239, 1979.

SZMANT, A. M. Nutrient enrichment on coral reefs: Is it a major cause of coral reef decline? Estuaries, v. 25, n. 4, p. 743-766, 2002.

TER BRAAK, C. J. F.; VERDONSCHOT, P. F. M. Canonical correspondence analysis and related multivariate methods in aquatic ecology. Aquat. Sci., v. 57, n. 3, p. 255-289, 1995.

VERMEIJ, M. J. A.; VAN MOORSELAAR, I.; ENGELHARD, S.; HÖRNLEIN, C.; VONK, S. M.; VISSER, P. M. The effects of nutrient enrichment and herbivore abundance on the ability of turf algae to overgrow coral in the Caribbean. PLoS ONE, v. 5, n. 12, p. e14312, 2010.

WESTNEAT, M. W.; ALFARO, M. E. Phylogenetic relationships and evolutionary of the reef fish family Labridae. Mol. Phylogenet. Evol., v. 36, n. 2, p. 370-390, 2005.

WILKINSON, C. Status of coral reefs of the world: 2008. Global Coral Reef Monitoring Network. Townsville: Australian Institute of Marine Science, 2008. 304 p.

WILSON, S. K.; GRAHAM, N. A. J.; POLUNIN, N. V. C. Appraisal of visual assessments of habitat complexity and benthic composition on coral reefs. Mar. Biol., v. 51, n. 3, p. 1069-1076, 2007.

ZAR, J. H. Biostatistical analysis. 4th ed. New Jersey: PrenticeHall, 1999. 663 p. 